\title{
Theoretical pulsation models for Long Period Variables
}

\author{
Y. Tuchman \\ Racah Institute of Physics, The Hebrew University of Jerusalem, \\ Jerusalem 91904, Israel
}

\begin{abstract}
Three issues, which are to my opinion the main obstacles in modeling the variability of Miras and Long Period Variables (LPVs), are presented and discussed:

a. The initial static models - Possible influences of the evolutionary details, the helium shell flashes and the use of the core-mass luminosity relation.

b. Modeling the convective energy transport - The mixing-length theory, its limitations and validity. First attempts for two-dimensional calculations of the convective flow in AGB stars.

c. The surface conditions - Introduction to the main difficulties and uncertainties. What should be done in the future.

Finally, the ancient problem, concerning the mode of pulsation, in Miras, is discussed using the recent radii observations confronted with the results of our full non-linear calculations.
\end{abstract}

\section{Major problems in modeling Long Period Variables}

\subsection{The initial static configurations (Wagenhuber \& Tuchman 1996)}

This problem which, in practice, is considered of a secondary importance, might be crucial in the understanding of the general features of Miras or Long Period Variables (LPVs) as a group. All previous initial models for LPVs have been created using the, well known, core-mass luminosity (CML) relation (Paczyński 1971; Becker \& Iben 1980). The real situation, however, is quite different. One should always remember that this core-mass luminosity (CML) relation was built for the evolutionary phase during which the star passes through the successive helium shell flashes. This relation was created to fit the luminosity around the peaks of the quiescent hydrogen-burning phase, between flashes.

The photospheric luminosity of a $1.5 M_{\odot}$ as a function of time is presented in Fig. 1 (Wagenhuber \& Tuchman 1996) for most of the relevant AGB phase. The core-mass (labeled $M_{C}$ ) as well as the luminosity, according to the CML relation $\left(L\left(M_{c}\right)\right.$, dotted line), are presented as well.

At this point we are not interested in the very rapid flash phase (of about 1000 years), even though, it might be very important as a trigger for mass loss (e.g. the creation of multiple shell planetary nebulae, Tuchman \& Barkat 1980). Concentrating upon the slow inter-flash phases (each of about $10^{5}$ years), the following conclusions should be drawn from Fig. 1: 


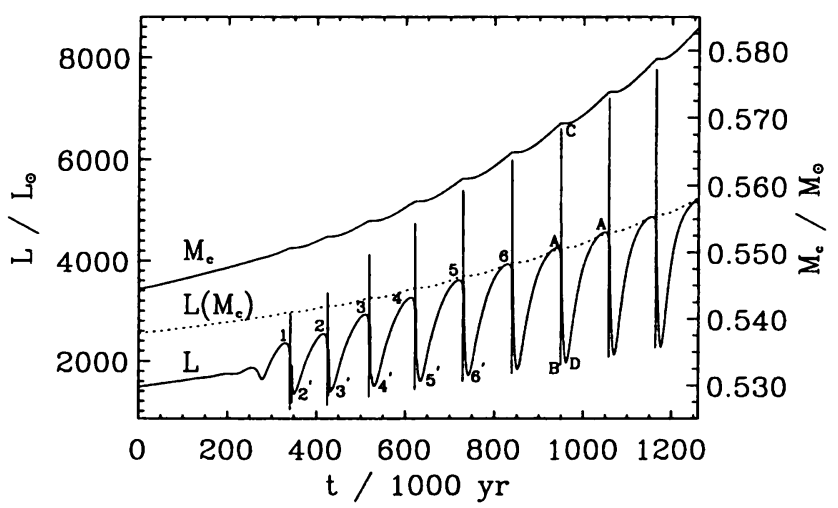

Figure 1. The photospheric luminosity evolution (L) of a $1.5 M_{\odot}$ star along the AGB. The dotted line $\left(L\left(M_{c}\right)\right)$ presents the commonly used evolution assuming the CML relation. Also shown is the evolution of the core mass $M_{c}$

a. The CML relation holds only for about $20 \%$ of the time. Elsewhere, in general, the real luminosity is lower than the one dictated by the CML relation.

b. The same luminosity can be obtained for a relatively large range of core masses (e.g. a luminosity of $2000 L_{\odot}$ is obtained in all inter-flash periods).

c. In the first few flashes the luminosity is much below the values given by the CML relation.

d. The stellar luminosity might change by a factor of 2 or even more during one inter-flash period.

All these facts should be considered in any attempt to confront theoretical models of these stars with observations.

The stellar periods, like its luminosity, are not monotonically increasing as might be predicted from the CML relation (Fig. 2). As it can be seen from Fig. 2, the linear periods might change by almost a factor of three during one inter-flash time interval.

The first apparent conclusion caused by the differences between the real evolution and the one dictated by the CML relation (Fig. 2) is the relatively fast period changes. In our specific example, the period of the fundamental mode in a $1.5 M_{\odot}$ star is increasing about 0.3 days per 100 years, to be compared with 0.02 days according to the CML relation. Much faster increase is expected for more massive cores $\left(>0.8 M_{\odot}\right)$, with progenitors of about $4 M_{\odot}$. In these cases a change of about 3 days per 100 years is expected (can it be confirmed with observations?).

A second conclusion is that stars might switch to a lower mode of pulsation while passing through the low luminosity range of the inter-flash periods. Stars located at these phases will probably appear as semi-regulars, in addition to the pre-flashes period.

A last important conclusion, derived from the real period evolution, is the possibility for a star with highly evolved core-mass (above $0.8 M_{\odot}$ ) to have rel- 


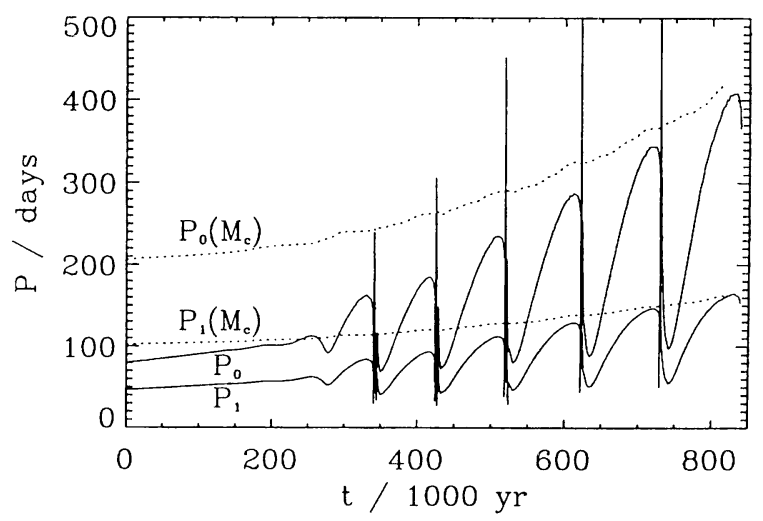

Figure 2. The period evolution, fundamental mode $\left(P_{0}\right)$ and first overtone $\left(P_{1}\right)$, calculated for the real luminosity sequence (full line) and for the one which assumes the CML relation (dotted line) as was presented in Fig. 1.

atively low luminosity, just starting an inter-flash period. This situation might explain the possibility to observe stars, with features characteristic for evolved stars, also in a region commonly believed to be occupied with low mass cores (low luminosity), as for example carbon stars (Lattanzio 1989).

\subsection{Modeling the convection in LPVs (Asida \& Tuchman 1997)}

An accurate calculation of the convective energy transport in envelopes of AGB stars is one of the crucial demands in any attempt for modeling these stars. Its importance is caused by the fact that the convective region in these stars is very efficient and large (in mass and radius).

The method commonly used to calculate the convective flux in AGB stars is the well-known mixing length theory (MLT, Bohm-Vitense 1958) and its variants (Baker \& Gough 1979). Four major defects are embedded in the MLT. The assumptions that the convective flux is: local, instantaneous, characterized by one-size eddies, and uses an unknown length-scale (the mixing-length parameter). Many attempts have been carried out in order to release one or more of these assumptions (e.g. Ulrich 1976; Stellingwerf 1982; Xiong, Cheng \& Deng 1997; Canuto \& Mazzitelli 1991). The success was always found to be only partial and in most cases the corrections were very complicated and difficult to be used. At this point a multidimensional ( $3 \mathrm{D}$ or at least $2 \mathrm{D}$ ) nonlinear simulation was naturally required.

Previous two-dimensional (2D) calculations have already been performed for RR-Lyrae stars (Deupree 1977, 1985). However, the convection zone in these stars is narrow and its efficiency is relatively low, so that the convection is a small perturbation to the radiative structure. In these cases, the needed relaxation time, to follow the star thermal changes, from an initial radiative structure to the convective one, is therefore short enough to be traced by a twodimensional calculation. This privilege does not exist in envelopes of AGB stars. 


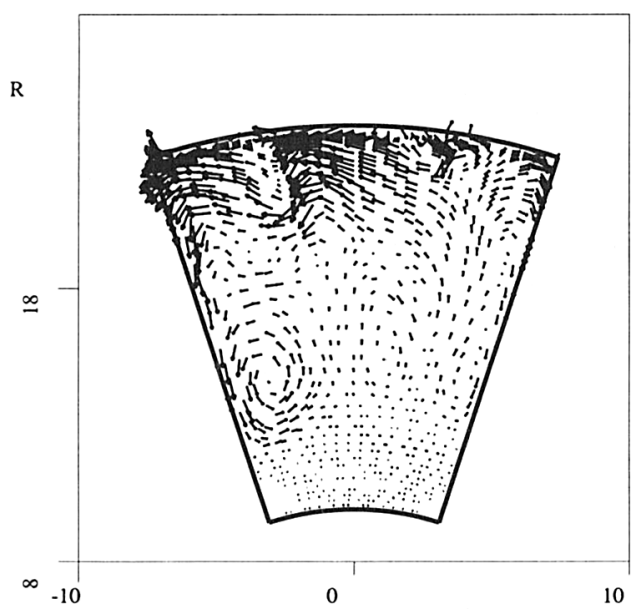

Figure 3. A typical velocity field of the convective flow in a $2 \mathrm{D}$ calculation

This problem, as will be explained below, practically eliminates the possibility for a simple direct calculation of the final $2 \mathrm{D}$ convective profile starting from an arbitrary initial configuration.

Two main time-scales are ruling the thermal relaxation of the stellar envelope at the AGB phase: the convective time-scale, i.e. the time required to reach a steady turbulence flow, which equals to several convective turnovers; and the thermal time-scale, i.e. the time needed for achieving thermal equlibirium.

The convective time-scale, for a typical AGB envelope, (the height of a convective cell divided by the mean convective velocity) is a few days. The thermal time-scale, on the other hand, is more than 50 years.

A typical time-step in a $2 \mathrm{D}$ code is about 100 seconds (mainly imposed by limitations of the relative energy changes). Each time-step needs about 1 second CPU time. Thus, for a typical AGB envelope, the convective flow evolves in a few CPU hours while the thermal relaxation takes about one year of CPU time. A full 2D simulation for an AGB envelope is therefore unrealistic with the present computational capabilities.

This difficulty was partially solved using the following procedure (Asida \& Tuchman 1997): two dimensional calculations were followed only for about one year - long enough for the establishment of the convective flow, but still far below the thermal time-scale needed for achieving full energy balance. However, this period of time was long enough to estimate, for each calculated model, its deviation from an accurate energy balance. Thus, using different initial envelope models (created with different values for the mixing-length parameter), we could approach the desired steady configuration. There is no way to ensure the convergence of this procedure, simply because it is based on the assumption that one of the initial models (integrated using the MLT) is indeed very close to the final 2D structure. However, if it does converge, it will obviously lead to the correct profile.

The typical structure of the turbulence flow is presented in Fig. 3. 


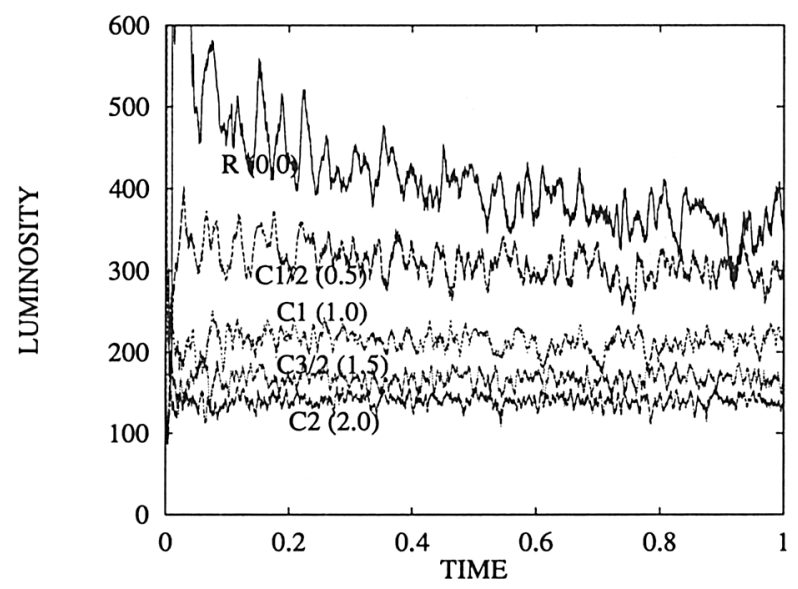

Figure 4. Outer luminosities in the 2D calculations for the different values of $\lambda(0 ., 0.5,1.0,1.5$ and 2.0$)$ used in calculating the initial profile (upper curve for minimal $\lambda$, etc.)

Fig. 4 shows the surface luminosity evolution in a star of $1.2 \mathrm{M}_{\odot}$ star, with a core of $0.96 \mathrm{M}_{\odot}$, where a luminosity of $200 L_{\odot}$ was assumed to enter into the envelope at its bottom. The surface luminosity evolution is shown for five different initial models which were integrated using the mixing-length theory with five different scale-lengths: $0,0.5,1 ., 1.5$ and 2 times the local pressure scale height. Since the luminosity that enters the envelope was constantly equal to $200 L_{\odot}$, it is clear from Fig. 4, that in those cases where the mixing-length parameter $(\lambda)$ differs from $\sim 1$ the envelope is far from thermal equilibrium.

In those cases, where $\lambda$ is smaller than 1 , the outer luminosity is found to be much above the luminosity that goes into the envelope $\left(200 L_{\odot}\right)$ and the envelope is loosing energy. In these cases one can recognize a relatively fast decrease in the outer luminosity (Fig. 4). For the models created initially with $\lambda$ larger than 1 , the surface luminosity is below $200 L_{\odot}$ and the envelope is accumulating energy. In these cases the change in the surface luminosity is too moderate to be recognized during a period of one year.

The similarity between the integrated 1D model, with $\lambda=1$, and the explicit 2D simulation, is not restricted only to the outer luminosity but also to its detailed structure. Fig. 5 presents, as an example, the local energy flux in both models.

More details about the 2D calculations, methods and assumptions, can be found in Asida \& Tuchman (1997).

The main conclusions that should be drawn from these preliminary $2 \mathrm{D}$ calculations are the following:

a. A static 1D model, integrated using the mixing-length theory for calculating the convective energy-flux, was found to be very similar with the exact $2 \mathrm{D}$ simulation. There is no proof that this result can be generalized to any convective envelope of an AGB star. Much more work is needed to clarify this point. 


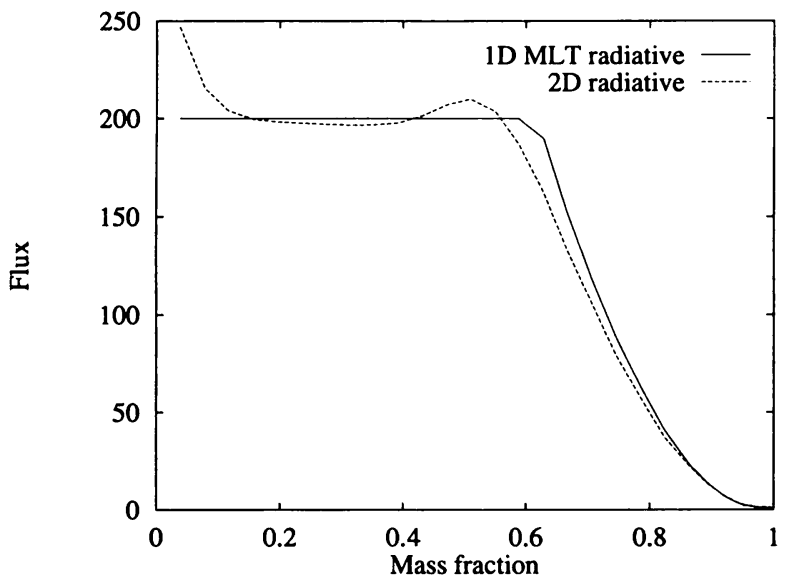

Figure 5. The detailed radiative luminosity, as a function of mass, for an AGB envelope, presented for the 2D converged model, compared with the best $1 \mathrm{D}$ integrated profile $(\lambda=1)$ (the convective luminosity= $200 L_{\odot}$-radiative flux)

b. The value of the mixing-length parameter $(\lambda)$ which was found to provide the best fit with the $2 \mathrm{D}$ calculations is one pressure scale-height. However, it should be emphasized that according to more recent $2 \mathrm{D}$ calculations, with much better space resolution, the best value for $\lambda$ is found to be close to 1.5 rather than 1.

It should be pointed that the results presented above have been obtained just for one model, at the beginning of the AGB phase. It is not clear, yet, whether the compatibility with the mixing length theory, on the whole, and the specific value fitted for the mixing-length parameter, are even nearly the same for other envelopes as well. This question should be clarified in the future.

The major drawback of the procedure we used for obtaining the final $2 \mathrm{D}$ configuration is that it can not be extended to dynamical calculations. Therefore, non-linear 2D calculations of the variability in Miras are still beyond our practical horizon.

\subsection{The surface conditions}

Unfortunately the modeling of pulsating LPVs is divided, for a long time, into the dynamical simulations of the envelope, on one side, and detailed atmospheric calculations on the other. This, unnatural, separation leads to the absence of adequate outer surface conditions for dynamical envelope simulations, and at the same time to a lack of reliable inner boundary conditions for the atmosphere calculations. In both cases synthetic prescriptions are used. For the envelope calculations one assumes a schematic dependence of the external pressure upon the surface radius, while, for the atmosphere calculations, a rigid piston moving with a dictated, time-dependent, velocity is assumed.

To my opinion, it is time for a combined (envelope + atmosphere) calculation. For the beginning, it might be sufficient to use very basic and simple 
methods, just in order to provide a reasonable evaluation for the mutual influence of these two adjacent media.

It is hard to guess the results of these combined calculations, but it is not unconceivable that it will lead to some considerable changes in the present theoretical estimates for the pulsating amplitude, spectra, mass loss and even for the non-linear periods of Miras and Long Period Variables.

\section{On the mode of pulsation in Miras - Non-linear calculations con- fronted with radii observations (Ya'ari \& Tuchman 1998)}

In spite of the huge difference (a factor of two and more), between the linear values of the fundamental mode and the first overtone in Miras, a clear identification of the dominant pulsation mode, in these stars, is still uncertain. The problem is partially caused by observational uncertainties, as well as by our relative ignorance in estimating the total mass of the observed variables. However, the main contribution is in modeling the convective energy transport. The major ambiguity, in our models, is the exact value used as a scale-length in the mixing-length method (Bohm-Vitense 1958) for calculating the convective flux. For example, it turns out that the linear period of the fundamental mode, calculated using the mixing length theory with one pressure scale-height as the mixing-length parameter $(\lambda)$, coincides with the first overtone period obtained for the same model but with $\lambda=1.6$ pressure scale-height. This theoretical ambiguity can be resolved if a reasonable estimate of the surface radii of these Mira stars could be observationally determined. The reason is that unlike the period-luminosity relation, which has been shown to be very sensitive to the details in modeling the convective flux, the period-radius relation is found to be almost independent of the specific way convection is being treated. The new direct measurements of Miras radii (Haniff, Scholz \& Tuthill 1995; van Belle et al. 1996; van Leeuwen et al. 1997) were very promising as a final decisive way to determine the actual mode of pulsation in these stars. Indeed, according to most of the investigations published since then, the first overtone is strongly favored as the dominant mode of pulsation (i.e. Haniff, Scholz \& Tuthill 1995; Feast 1996; van Leeuwen et al. 1997).

To our opinion, all previous attempts confronting radii observations of Miras with the theoretical predictions, in order to determine their pulsation mode, have neglected two crucial facts that might invert their conclusions:

a. The observed data has always been compared to the theoretical linear values. Whereas the nonlinear periods, as well as the nonlinear radii, are found to be very different (Ya'ari \& Tuchman 1996). Changes up to $30 \%$ should be expected.

b. All previous studies have ignored the variability in the surface radius caused by the stellar pulsation. Actually, it was assumed to be very small (less than 10\%, Haniff, Scholz \& Tuthill 1995). The exact phase at which the observation is performed was therefore ignored.

The incorrectness of this assumption is easily shown using the observed velocities in Mira variables; if $\Delta \mathrm{V}$ is the observed velocity amplitude, the corresponding change in the surface radius, during one period $(P)$, can roughly (assuming a linear approximation) be estimated to $(1 / 2) \Delta V P$. For a typi- 


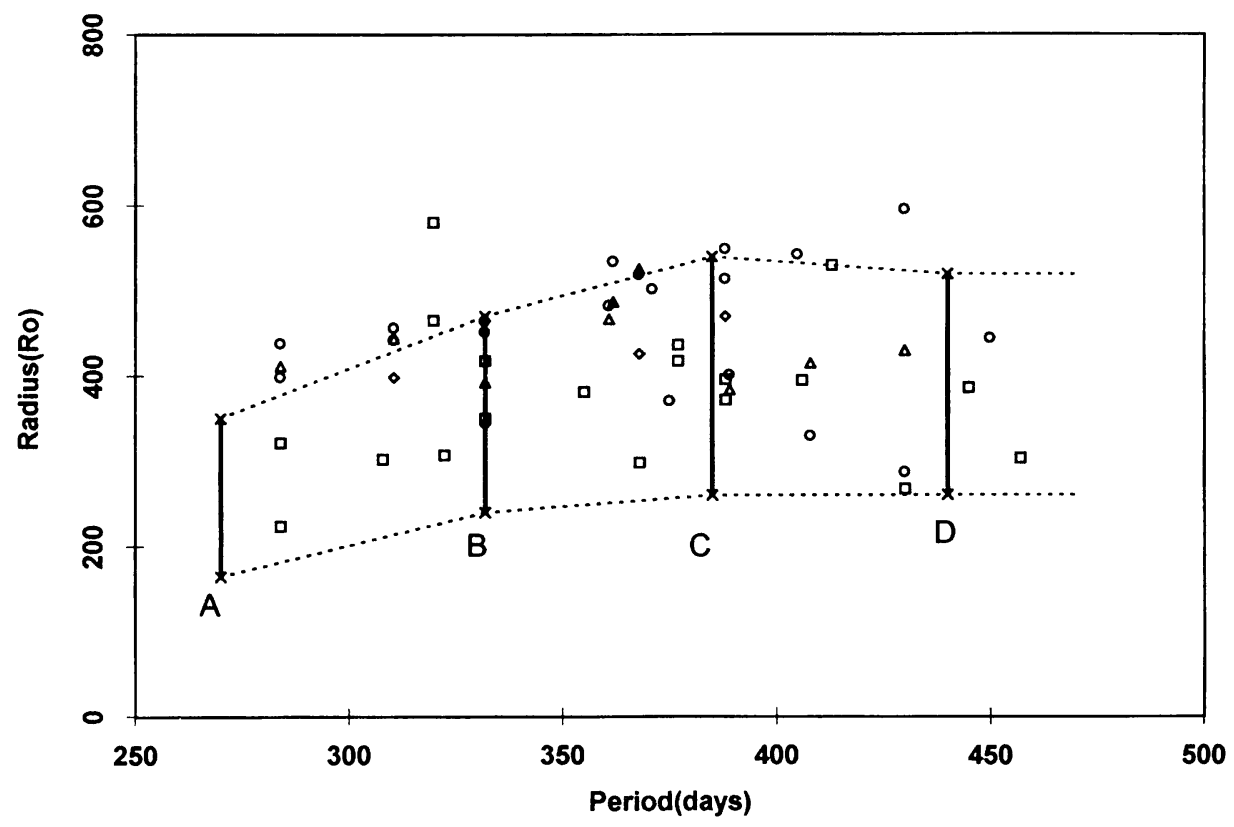

Figure 6. Effective observed radii, as a function of period, accumulated from the following sources: van Leeuwen, Feast, Whitelock \& Yudin 1997 (circles), Feast 1996 (diamonds), Haniff, Scholz \& Tuthill 1995 (triangles) and van Belle, Dyck, Benson \& Lacasse 1996 (squares). The pulsational variability in the surface effective radii for the four chosen calculated models (labeled A-D) is shown as well

cal Mira $\Delta V=20 \mathrm{~km} / \mathrm{sec}$ (Hinkle 1978; Hinkle, Scharlach \& Hall 1984) and $P=300$ days. Thus, the whole change in the surface radius, during one period, is about 400 solar radii. This is obviously a crude estimate but it is good enough to demonstrate the fact that the radius variability can not be ignored when comparing the observations with theory.

In the following, a detailed comparison between the observed radii of Mira variables and the theoretical non-linear calculated values, taking into account the two remarks above, is described.

Observed radii of Mira variables have been compiled from four different sources (van Leeuwen et al. 1997; Feast 1996; Haniff, Scholz \& Tuthill 1995; van Belle et al. 1996). This data is presented in Fig. 6. Four theoretical models (with masses between 1.2 and $1.5 M_{\odot}$ ) covering the relevant period range, have been selected, to be compared with this observed data. Their pulsational variability in the surface effective radii, minimum to maximum, are also marked in Fig. 6.

As can be seen from Fig. 6 most of the observed points are located between the boundaries connecting the minimum and maximum theoretical nonlinear radii of the Mira models pulsating in their fundamental mode. Only few observed 
radii are located above, but within the observational error of the theoretical range.

We may therefore conclude, in contrast to previous works, that the radii observations of Miras are compatible with the theoretical non-linear models pulsating in the fundamental mode.

\section{References}

Asida S.M., Tuchman Y., 1997, ApJ 491, L47

Baker N.H., Gough D.O., 1979, ApJ 234, 232

Becker S.A., Iben I., Jr., 1980, ApJ 237, 111

Bohm-Vitense E., 1958, Zs. f. Astrophysik 46, 108

Canuto V.M., Mazzitelli I., 1991, ApJ 370, 295

Deupree R.G., 1977, ApJ 211, 509

Deupree R.G., 1985, ApJ 296, 160

Feast M.W., 1996, MNRAS 278, 11

Haniff C.A., Scholz M., Tuthill P.G., 1995, MNRAS 212, 783

Hinkle K.H., 1978, ApJ 220, 210

Hinkle K.H., Scharlach W.W.G., Hall D.N.B., 1984, ApJS 56, 1

Lattanzio J.C., 1989, in Evolution of Peculiar Red Giant Stars, IAU Coll. 106, Cambridge University Press, p. 161

Paczyński B., 1971, Acta Astr. 21, 417

Stellingwerf R.F., 1982, ApJ 262, 330

Tuchman Y., Barkat Z., 1980, ApJ 242, 199

Ulrich R.K., 1976, ApJ 207, 564

van Belle G.T., Dyck H.M., Benson J.A., Lacasse M.G., 1996, AJ 114, 2150

van Leeuwen F., Feast M.W., Whitelock P.A., Yudin B., 1997, MNRAS 287, 1027

Wagenhuber J., Tuchman Y., 1996, A\&A 309, 511

Xiong D.R., Cheng Q.L., Deng L., 1997, ApJS 108, 529

Ya'ari A., Tuchman Y., 1996, ApJ 456, 350

Ya'ari A., Tuchman Y., 1998, ApJ, submitted 


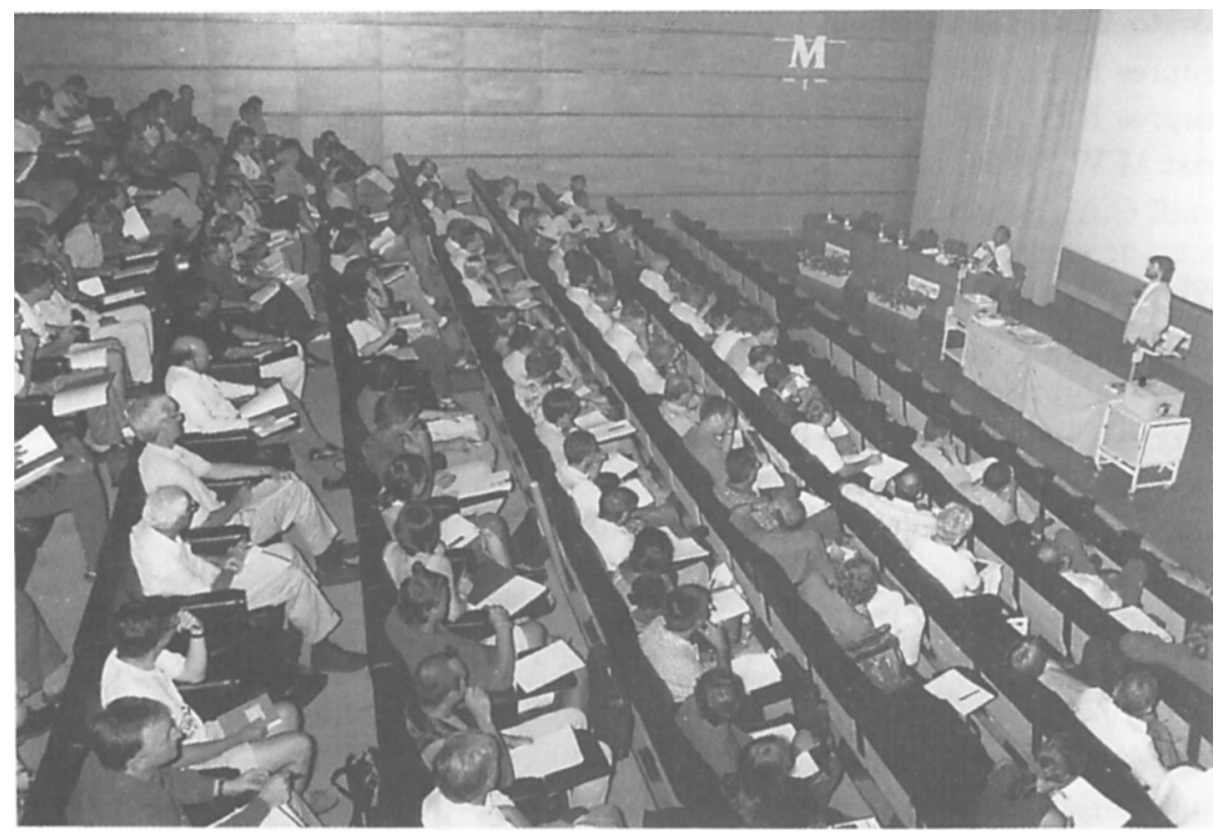

The audience during one of the lively discussions (here with Thomas Blöcker) 